\title{
Correlação entre posicionamento escapular, análise postural funcional e grau de incapacidade dos membros superiores (DASH Brasil) em médicos ultrassonografistas*
}

\author{
Correlation among scapular positioning, functional postural analysis and upper extremities \\ disability degree in sonographers (DASH Brazil) \\ Geraldo Fabiano de Souza Moraes ${ }^{1}$, Fernanda Moreira Gonçalves ${ }^{2}$, Júnia Darth Silva ${ }^{2}$, Natália \\ Spindola Soares ${ }^{2}$
}

Resumo OBJETIVO: Avaliar e mensurar o posicionamento escapular e correlacioná-lo com o grau de incapacidade e avaliação funcional de ultrassonografistas. MATERIAIS E MÉTODOS: Dezoito médicos, divididos em grupos sintomático e assintomático em relação à presença de dor por mais de seis meses, responderam a uma entrevista e foram submetidos a avaliação física e aplicação do DASH Brasil. Foi realizada a medida do ângulo de inclinação escapular e avaliação do posicionamento dos membros superiores durante a ultrassonografia. Foram utilizados testes $U$ Mann-Whitney para avaliação do questionário de incapacidade, $t$ de Student para posicionamento do membro superior, ANOVA para inclinação escapular e coeficiente de Spearman para correlação do posicionamento funcional de membros superiores e grau de incapacidade. RESULTADOS: Nas 30 primeiras questões do DASH Brasil houve diferença significativa entre os grupos sintomático e assintomático. No módulo opcional, relacionado ao trabalho, houve diferença significativa entre os grupos. As medidas do inclinômetro apresentaram diferenças significativas entre os grupos no plano frontal em $90^{\circ}$ e $120^{\circ}$. Para o ângulo funcional dos membros superiores não houve diferença significativa entre os grupos. CONCLUSÃO: Alterações no ângulo de inclinação escapular podem levar à disfunção dos membros superiores e a aplicação do DASH Brasil pode indicar o grau de dor e incapacidade do indivíduo.

Unitermos: Biomecânica; Complexo articular do ombro; Posicionamento escapular; Ritmo escapuloumeral; Médico ultrassonografista.

Abstract OBJECTIVE: To evaluate and measure the scapular positioning, and establishing its correlation with functional postural analysis and degree of upper extremities disability in sonographers. MATERIALS AND METHODS: Eighteen physicians divided into two groups - symptomatic individuals with pain for more than six months, and asymptomatic individuals - were interviewed and submitted to physical assessment with application of the Brazilian version of the DASH questionnaire. Functional measurements of scapular inclination angles and upper limbs positioning were obtained during the ultrasound scanning. The Mann-Whitney $U$ test was utilized for analyzing the disability questionnaires; the $t$-Student test, for upper limbs positioning; ANOVA, for scapular inclination angles, and the Spearman's test for correlation between the functional upper limbs positioning and disability degree. RESULTS: A significant difference between symptomatic and asymptomatic groups was observed in the first 30 items of the DASH questionnaire as well as in the four study-related questions. Measurements of scapular positioning presented significant differences between the groups in the frontal plane at $90^{\circ}$ and $120^{\circ}$. No significant difference was found for the analysis of functional upper limbs positioning. CONCLUSION: Changes in the scapular positioning may result in upper limbs dysfunction, and the application of the DASH questionnaire can indicate the level of pain and disability of an individual.

Keywords: Biomechanics; Shoulder joint complex; Scapular positioning; Scapulohumeral rhythm; Sonographer.

Moraes GFS, Gonçalves FM, Silva JD, Soares NS. Correlação entre posicionamento escapular, análise postural funcional e grau de incapacidade dos membros superiores (DASH Brasil) em médicos ultrassonografistas. Radiol Bras. 2009;42(1):31-36.

* Trabalho realizado no Centro Universitário Newton Paiva, Belo Horizonte, MG, Brasil.

1. Mestre, Fisioterapeuta, Professor Adjunto e Coordenador do Curso de Fisioterapia do Centro Universitário Newton Paiva, Belo Horizonte, MG, Brasil.

2. Fisioterapeutas, Bacharéis em Fisioterapia pelo Centro Universitário Newton Paiva, Belo Horizonte, MG, Brasil.

Endereço para correspondência: Dr. Geraldo Fabiano de Souza Moraes. Avenida Silva Lobo, 1718, sala 11011, andar térreo, Nova Granada. Belo Horizonte, MG, Brasil, 30480-230. E-mail: geraldmoraes@terra.com.br

Recebido para publicação em 25/6/2007. Aceito, após revisão, em 20/8/2008.

\section{INTRODUÇÃO}

Atualmente, há uma grande preocupação em prevenir doenças e lesões que envolvem a articulação do ombro decorrentes de alterações posturais que levam a compensações do sistema em detrimento da funcionalidade ${ }^{(\mathbf{1})}$.
A prevalência de dor no ombro, acompanhada de incapacidade, está presente em cerca de $20 \%$ na população em geral $^{(2)}$. As causas de sobrecarga nesse complexo articular são multifatoriais, tais como: grande repetitividade dos movimentos, posicionamento do membro superior, principalmente em abdução do ombro, fadiga muscular, 
fatores de organização do trabalho e anulação do mecanismo de regulação do trabalho, como sobrecarga (diminuição ou falta de intervalo), e fatores psicossociais, que acarretam tensão e estresse ${ }^{(3)}$.

Tem-se notado a importância da percepção na saúde e qualidade de vida de grande parte da população, que é prejudicada, frequentemente, por distúrbios músculo-esqueléticos agudos e crônicos ${ }^{(\mathbf{4})}$.

O movimento escapular, juntamente com a movimentação do úmero, permite uma amplitude de movimento de flexão ou abdução do braço de $150^{\circ}$ a $180^{\circ}$. A mobilidade típica em indivíduos assintomáticos geralmente ocorre com dois graus de movimento glenoumeral para um grau de movimento escapular, o que determina uma relação de 2:1 no ritmo escapuloumeral ${ }^{(\mathbf{5 , 6})}$.

Diversos atletas, principalmente nadadores $^{(7)}$, e profissionais que atuam com o membro superior abduzido têm predisposição maior para desenvolver alterações na relação mobilidade-estabilidade ${ }^{(\mathbf{8})}$. Por exemplo, a atividade profissional do médico ultrassonografista é caracterizada pela alta probabilidade de ocorrer alteração da relação mobilidade-estabilidade devido à permanência de longos períodos na posição de abdução ${ }^{(\mathbf{9}, 10)}$.

O diagnóstico por ultrassonografia é atividade médica relativamente nova, que existe desde o início de $1940^{(9)}$. Com o advento de novas tecnologias e o reconhecimento da capacidade diagnóstica do ultrassom, tem aumentado, a cada ano, o número e a duração na execução dos exames de ultrassonografia ${ }^{(\mathbf{9}, 10)}$. Apesar do avanço tecnológico ter permitido obter imagens de alta resolução, as condições ergonômicas para a realização dos exames não são nada favoráveis ao sistema músculo-esquelético do médico ultrassonografista ${ }^{(11)}$.

Aproximadamente $80 \%$ desses profissionais relatam desconforto ou incapacidade de realizar um exame adequado, pela presença de sintomas nos membros superiores. Um dos fatores que parecem interferir na origem dos sintomas do médico ultrassonografista é o alto número de exames de rotina que passou a ser efetuado, além de riscos como choques elétricos, baixo índice de iluminação, estresse e sobrecarga músculo-esquelética nos membros superiores e na coluna vertebral ${ }^{(2,12)}$.
As sobrecargas estáticas e dinâmicas impostas na musculatura do cíngulo escapular e da extremidade superior são devidas às necessidades de manutenção do ombro em abdução para uma melhor função do punho e mão, durante a fixação do transdutor perpendicularmente à pele do

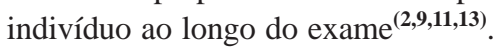

O comprometimento na funcionalidade e o afastamento do indivíduo de suas atividades cotidianas levam a uma sobrecarga dos sistemas de saúde nos diversos países. É importante que medidas preventivas sejam desenvolvidas e, para isso, é necessário identificar e entender a sobrecarga da atividade ocupacional e seus efeitos, para se minimizar as consequências decorrentes do comprometimento funcional do indivíduo $^{(\mathbf{1})}$. Embora se reconheça o adoecimento relacionado ao trabalho como carga tridimensional (física, psíquica e cognitiva), os objetivos deste estudo foram avaliar e mensurar o posicionamento escapular e correlacioná-lo com o grau de incapacidade dos membros superiores e análise postural funcional de ultrassonografistas, centrando-se na carga física de trabalho.

\section{MATERIAIS E MÉTODOS}

\section{Delineamento do estudo e amostra}

Estudo transversal, aprovado pelo Comitê de Ética e Pesquisa do Centro Universitário Newton Paiva ( $\left.n^{\circ} 40 / 2005\right)$.

Participaram do estudo 18 voluntários, sendo nove do grupo sintomático e nove do grupo assintomático, sedentários, idade entre 27 e 52 anos. Todos os médicos realizavam ultrassonografia abdominal. O tempo de trabalho variou de 18 meses a 20 anos e a quantidade de exames realizados por dia variou de 10 a 30 . Os 18 participantes tiveram ambos os ombros avaliados (36 ombros). Dos indivíduos do grupo sintomático, cinco eram mulheres $(55,6 \%)$ e quatro eram homens $(44,4 \%)$, com média de idade de 33,67 $\pm 8,89$ anos (27 a 52 anos), massa corporal de $69,66 \pm 13,10 \mathrm{~kg}(57,0$ a 95,0 $\mathrm{kg})$ e estatura de $1,73 \pm 0,07 \mathrm{~m}$ (1,63 a 1,83 $\mathrm{m})$. A média do índice de massa corporal da amostra foi de $22,97 \pm 3,5 \mathrm{~kg} / \mathrm{m}^{2}(19,14$ a $31,04 \mathrm{~kg} / \mathrm{m}^{2}$ ). Dos participantes do grupo assintomático, quatro eram mulheres $(44,4 \%)$ e cinco eram homens $(55,6 \%)$, com média de idade de $38,78 \pm 7,79$ anos (30 a
49 anos), massa corporal de 76,11 $\pm 22,7$ $\mathrm{kg}(49,0$ a $115,0 \mathrm{~kg})$ e estatura de 1,69 \pm $0,07 \mathrm{~m}$ (1,54 a 1,85 m). A média do índice de massa corporal da amostra foi de 26,17 $\pm 6,5 \mathrm{~kg} / \mathrm{m}^{2}\left(18,9\right.$ a $\left.38,46 \mathrm{~kg} / \mathrm{m}^{2}\right)$.

\section{Inclinômetro digital}

Foi utilizado inclinômetro Mitutoyo Digital Protractor (Mitutoyo Evaluation Instruments; Chicago, IL) para mensuração do posicionamento escapular. A este foram adaptados dois braços de extensão $(10,0 \mathrm{~cm}$ cada), confeccionados em acrílico, para a correta acomodação na escápula e leitura dos graus de inclinação. Foi acoplado nível de água perpendicular ao equipamento, para garantir o correto alinhamento deste em relação aos planos de avaliação (Figura 1). A validade e a confiabilidade da utilização desse inclinômetro digital, como forma de medida do posicionamento escapular, foram descritas por Johnson et al. ${ }^{(6)}$, que usaram o mesmo instrumento e medidas.

\section{Questionário de incapacidades do braço, ombro e mão do Brasil}

Foi utilizado o questionário Disability Arm Shoulder Hand do Brasil (DASH Brasil), adaptado transculturalmente ao português do Brasil por Orfale et al. ${ }^{(14)}$. Esse questionário, elaborado originariamente no idioma inglês e denominado DASH Questionnaire, foi desenvolvido por Hudak et al. em 1996, com o objetivo de mensurar a incapacidade física e sintomas dos membros superiores em uma população heterogênea. Além disso, foi proposto para avaliar incapacidade e sintomas em uma única ou várias afecções dos membros superiores.

\section{Avaliação do ângulo funcional do membro superior}

Para a medida do ângulo funcional do membro superior, marcadores cutâneos confeccionados em acrílico foram colocados nas referências anatômicas, utilizando-se fita dupla face: sétima vértebra cervical (C7), sétima vértebra torácica (T7), acrômio e epicôndilo lateral. O registro da imagem do posicionamento funcional foi feito por meio de uma câmera fotográfica digital Sony Mavica MVC-FD 200 (Sony Electronics, Inc.; San Diego, CA).

Os dados foram observados na posição de repouso do membro superior (posição 
anatômica), a $30^{\circ}, 60^{\circ}, 90^{\circ}$ e $120^{\circ}$ de elevação do ombro nos planos frontal, escapular e sagital. A posição de avaliação adotada foi a mais frequentemente utilizada na realização do exame de ultrassom.

\section{Coleta de dados}

Inicialmente, todos os voluntários foram esclarecidos quanto aos objetivos e procedimentos e assinaram o termo de consentimento livre e esclarecido. Em seguida, um único avaliador, devidamente treinado, realizou uma entrevista individual, uma avaliação física e a aplicação do questionário DASH Brasil com todos os participantes. Os dados foram armazenados para posterior análise.

Com o indivíduo sentado, um segundo avaliador, devidamente treinado, posicio- nou o inclinômetro digital na espinha da escápula. Os braços adaptados ao equipamento foram acoplados à espinha da escápula na sua borda medial e inferior ao acrômio para o correto posicionamento e acomodamento do equipamento à referência anatômica (Figura 2). Um terceiro avaliador realizou a leitura e o registro dos valores observados na posição de repouso do membro superior (posição anatômica), a $30^{\circ}, 60^{\circ}, 90^{\circ}$ e $120^{\circ}$ de elevação do ombro nos planos frontal, escapular e sagital. Para garantir o posicionamento correto do membro superior nesses planos, foi utilizado um anteparo para normalização do posicionamento do membro superior durante a execução do exame.

Em seguida, marcadores cutâneos foram posicionados nas referências anatômi- cas para o registro fotográfico do ângulo funcional do posicionamento do membro superior durante a execução do exame de ultrassonografia. O voluntário assumiu a posição funcional de avaliação durante a simulação do exame de ultrassonografia. Foi feito o registro da posição do membro superior, orientando o voluntário a assumir a posição mais frequentemente utilizada na realização dos exames. As imagens foram armazenadas, e utilizando-se o programa AutoCAD 2004 (Autodesk, Inc.; San Rafael, EUA) avaliou-se o posicionamento do membro superior. Foram traçadas linhas de união dos pontos de C7 a T7 e do acrômio ao epicôndilo lateral. O ângulo funcional de posicionamento do membro superior para o exame foi determinado pelo ângulo formado por essas duas retas (Figura 3).

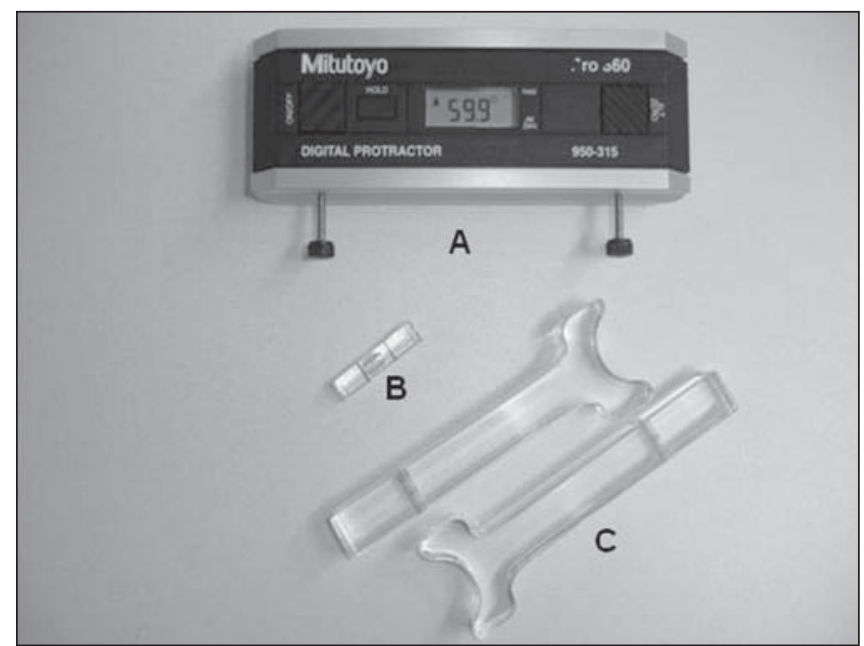

Figura 1. Material utilizado para medida do ângulo de inclinação escapular. A, inclinômetro digital; $B$, nível de água; $C$, braços de extensão.

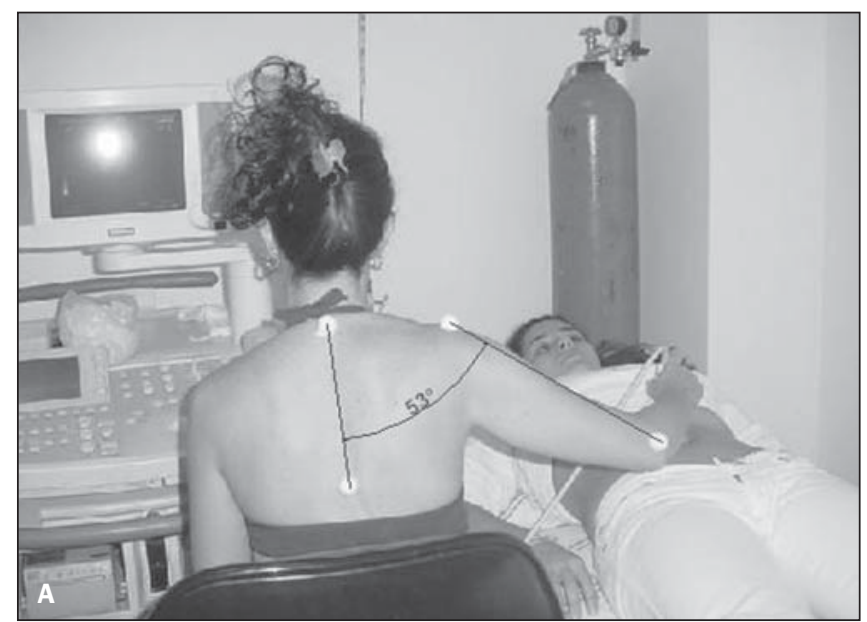

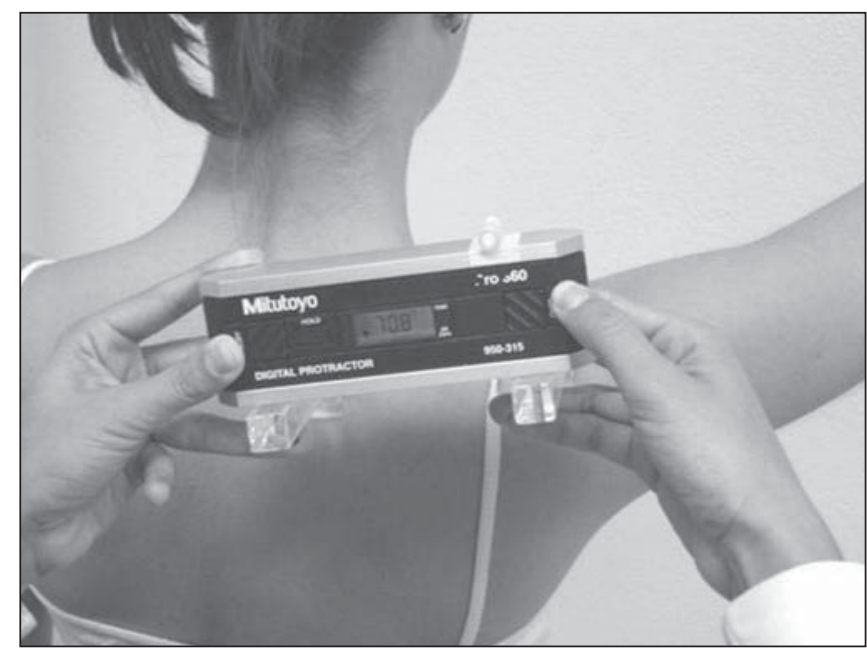

Figura 2. Posicionamento do inclinômetro digital para medida do ângulo de inclinação escapular.

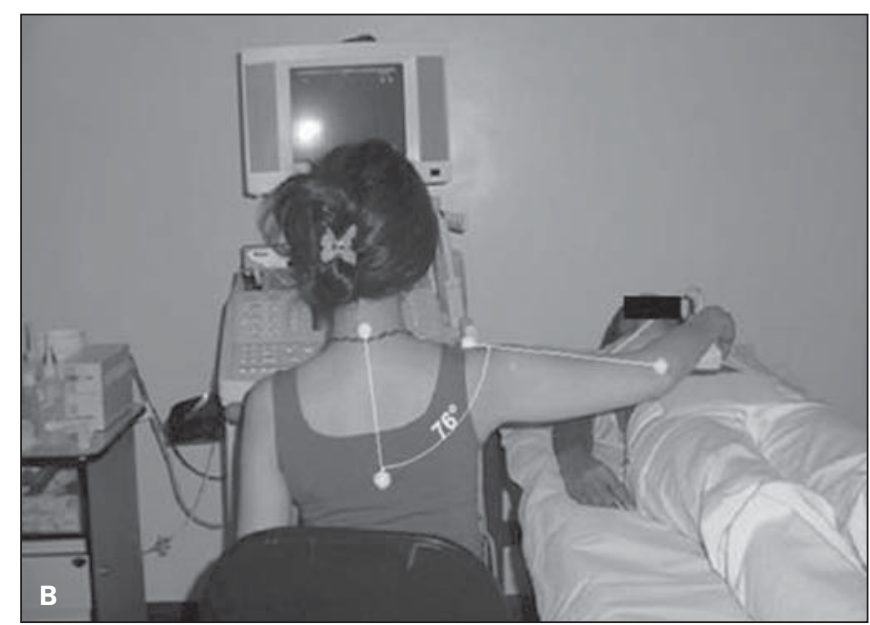

Figura 3. Ângulo funcional do membro superior, posições assintomática (A) e sintomática (B). 


\section{Análise estatística}

Estatísticas descritivas, testes de normalidade (Shapiro-Wilk) e testes de igualdade de variância (Levene) foram realizados para todas as variáveis, utilizando o pacote estatístico SPSS. Análise de variância (ANOVA) foi utilizada para investigar diferenças estatisticamente significantes entre os grupos e entre os lados para todas as variáveis, ângulo de inclinação escapular e avaliação funcional dos membros superiores. Teste $t$ de Student foi utilizado para comparar os valores encontrados no DASH Brasil. Coeficiente de Spearman foi utilizado para determinar se havia correlação na avaliação do posicionamento dos membros superiores e grau de incapacidade. O nível de significância estabelecido foi de $\alpha=0,05$.

\section{RESULTADOS}

A média de tempo de profissão dos indivíduos do grupo assintomático foi de dez anos e do grupo sintomático foi de quatro anos e nove meses. A média de exames por dia no grupo assintomático foi de 17,8 e no grupo sintomático foi de 22,7. Não houve diferenças estatisticamente significantes entre o tempo de profissão $(p=0,092)$ e o número de exames por dia $(p=0,186)$, comparando-se os grupos sintomático e assintomático.

\section{Variáveis analisadas}

DASH Brasil - Houve diferença estatisticamente significante para as primeiras 30 questões ( $p=0,001)$ e para o módulo opcional de trabalho ( $p=0,012)$, na comparação do grupo sintomático com o grupo assintomático, como ilustrado nas Figuras 4 e 5, respectivamente. A média das primeiras 30 questões do DASH Brasil, para o grupo sintomático, foi de 16,16 $\pm 13,18$ pontos (2,5 a 45,5 pontos) e das quatro opcionais, relacionadas ao trabalho, foi de $27,08 \pm 20,96$ pontos ( 0 a 56,25 pontos). A média das primeiras 30 questões do DASH Brasil, para o grupo assintomático, foi de $1,11 \pm 1,81$ pontos (0 a 5 pontos) e das quatro opcionais relacionadas ao trabalho foi de 4,16 $\pm 8,83$ pontos ( 0 a 25 pontos).

Ângulo de inclinação escapular Houve diferença estatisticamente significante de $6,65^{\circ}(p=0,016)$ e de $5,87^{\circ}(p=$
0,033) do ângulo de inclinação escapular no plano frontal a $90^{\circ}$ e $120^{\circ}$, respectivamente, comparando-se o ombro direito do grupo sintomático e o ombro direito do grupo assintomático. Observou-se, também, diferença estatisticamente significante de $4,43^{\circ}$ ( $\left.p=0,028\right)$ no grupo assintomático, comparando-se o ombro esquerdo em relação ao ombro direito, em repouso (0), no plano frontal.

Ângulo funcional do membro superior - Conforme ilustrado na Figura 6, o posicionamento funcional do membro superior não apresentou diferença estatisticamente significante entre os grupos sintomático e assintomático $(p=0,765)$, com

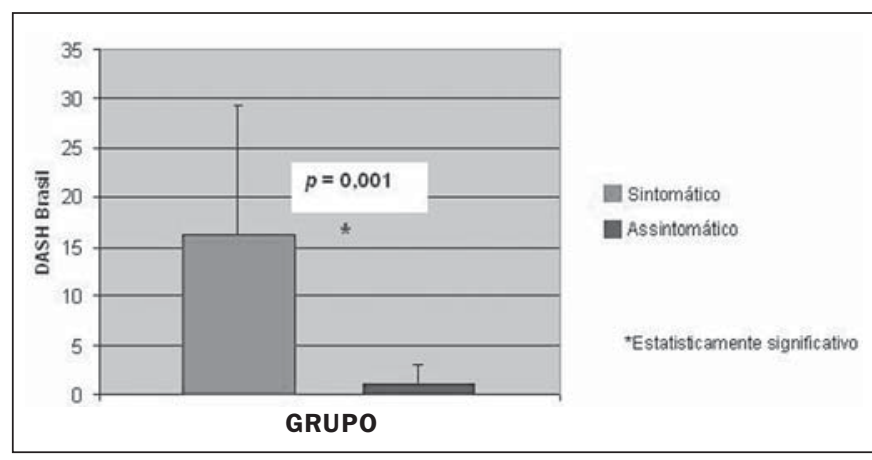

Figura 4. Comparação do grau de incapacidade avaliado pelo DASH Brasil entre os grupos sintomático e assintomático (médias e desvios-padrão).

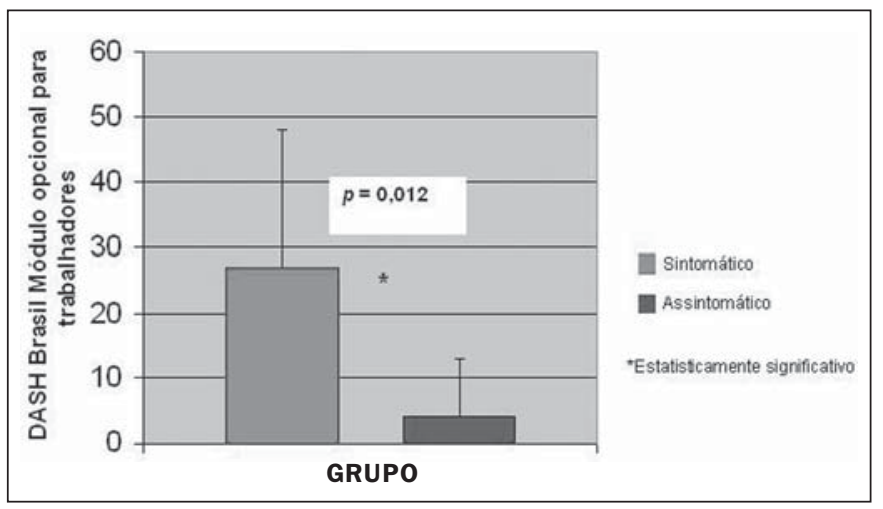

Figura 5. Comparação do grau de incapacidade avaliado pelo DASH Brasil, módulo opcional para trabalhadores, entre os grupos sintomático e assintomático (médias e desvios-padrão).

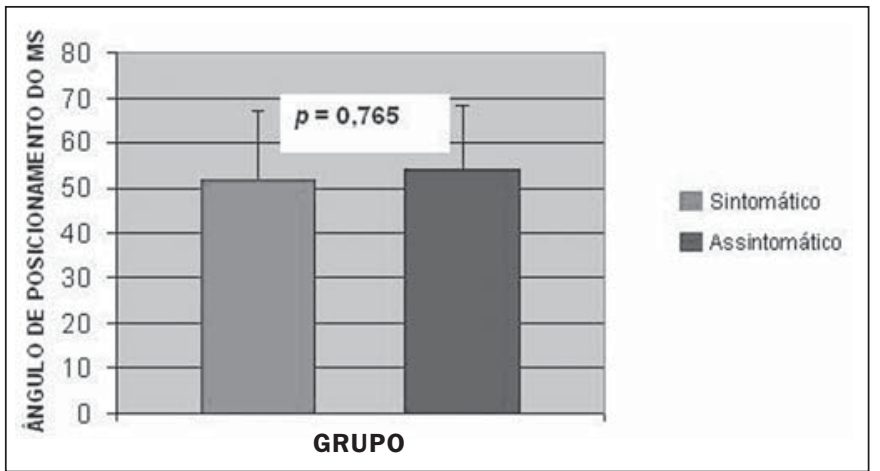

Figura 6. Comparação do ângulo de posicionamento do membro superior entre os grupos sintomático e assintomático (médias e desvios-padrão). 


\section{DISCUSSÃO}

Neste estudo não houve diferenças significativas entre o tempo de profissão e o número de exames realizados por dia, comparando-se o grupo sintomático e o grupo assintomático. Dessa forma, não houve relação direta entre o tempo de trabalho e os sintomas. Pode-se inferir que isto pode ter ocorrido graças a uma adaptação fisiológica da musculatura decorrente das necessidades da atividade ocupacional.

Por meio da avaliação pelo questionário DASH Brasil, as 30 primeiras questões apresentaram diferenças significativas entre os grupos sintomático e assintomático. Neste estudo, os resultados obtidos com o questionário indicaram dor e/ou desconforto no ombro dos indivíduos do grupo sintomático. Pode-se inferir que esses sintomas são decorrentes das posturas de abdução sustentada do ombro do médico ultrassonografista adotadas para a realização do exame, corroborados pela mensuração do ângulo de posicionamento do membro superior durante a realização do exame.

A necessidade de segurar o transdutor em abdução e sem apoio, principalmente para visualização de órgãos que se encontram no dimídio esquerdo do paciente, e o tempo prolongado da realização do exame de ultrassom apontam para uma relação direta com os sintomas relatados pelos voluntários deste estudo.

De acordo com os resultados do questionário DASH Brasil, foi possível, ainda, estabelecer um paralelo com os domínios da Classificação Internacional de Funcionalidade, Incapacidade e de Saúde (CIF). Foi observado que a atividade e participação no grupo sintomático demonstrou-se prejudicada devido à própria alteração das estruturas e funções do membro superior, que podem ter levado a um certo grau de incapacidade na realização tanto da atividade ocupacional quanto das atividades da vida diária. Entretanto, a incapacidade deve sempre ser vista dentro de um contexto biopsicossocial, uma vez que todos esses fatores interferem, diretamente, nos componentes da $\mathrm{CIF}^{(\mathbf{1 5}, 16)}$.

No presente estudo, o ângulo de posicionamento do membro superior durante a simulação do exame de ultrassonografia não apresentou diferenças significativas entre os grupos sintomático e assintomático. Foi observado que, na maioria das clínicas de ultrassonografia visitadas, os equipamentos utilizados para realização do exame seguiam uma certa padronização em relação à altura da cadeira do médico, altura da maca do paciente e distância do monitor. Essa padronização não é o ideal, uma vez que cada profissional tem um biótipo, e este fator pode ter interferido na angulação de abdução entre os indivíduos.

Jakes $^{(17)}$ e Muir et al. ${ }^{(18)}$ demonstraram que a postura adotada do membro superior para realização da atividade ocupacional representa um indicador de sobrecarga e risco de desenvolvimento de distúrbios do ombro. Jakes ${ }^{(17)}$ relata que as principais causas de lesão músculo-esquelética em médicos ultrassonografistas são a necessidade de manutenção do ombro em abdução para um melhor posicionamento do transdutor na pele do paciente, a realização do exame associado ao manuseio do monitor de imagens, e as condições ergonômicas como altura e direção do monitor, altura da cadeira do ultrassonografista e altura da maca para acomodação do paciente durante a realização do exame. Todos esses fatores, associados ao reduzido tempo de intervalo entre um exame e outro, podem contribuir para alterações funcionais e incapacidade permanente $^{(12,18,19)}$.

Considera-se aceitável, para trabalhos contínuos e de pequena sobrecarga, uma abdução média de $20^{\circ(15)}$. Uma vez que as médias encontradas no presente estudo superaram os $20^{\circ}$ recomendados, o risco do desencadeamento de desequilíbrios musculares, bem como alterações posturais e sobrecarga dinâmica, são, aparentemente, elevadas para os indivíduos avaliados no presente estudo.

Quanto às medidas do inclinômetro, diferenças significativas foram encontradas entre os grupos sintomático e assintomático no plano frontal, em $90^{\circ} \mathrm{e} 120^{\circ}$. A diferença observada em $90^{\circ}$ foi de $6,65^{\circ}$ no ombro direito dos voluntários sintomáticos em relação ao ombro direito dos assintomáticos, e em $120^{\circ}$ foi encontrada diferença de $5,87^{\circ}$ do ombro direito dos indivíduos sintomáticos em relação ao ombro direito dos assintomáticos. Houve, também, diferença significativa no grupo assintomático, no plano frontal, onde, em repouso (posi- ção 0 ), houve diferença de $4,43^{\circ}$ do ombro esquerdo em relação ao direito.

Ao permanecer com o ombro abduzido, em aproximadamente $90^{\circ}$, a musculatura pode sofrer fadiga, com consequente incapacidade de manutenção dessa posição por longos períodos, relacionada à atividade ocupacional (ultrassonografia). Tal fator pode levar à adaptação muscular, a fim de se garantir a funcionalidade; ademais, isso pode desencadear desequilíbrios musculares em arcos de movimentos em que ocorre uma dificuldade de grupos musculares manterem a adequada estabilização ou posicionamento de segmentos corporais durante a realização de uma atividade específica, como gestos esportivos e atividades ocupacionais.

Barbosa et al. ${ }^{(11)}$ afirmaram que durante a realização do exame o médico ultrassonografista necessita manter o ombro em abdução sem apoio, o que causa contração isométrica dos músculos do membro superior, principalmente do cíngulo escapular, na tentativa de promover uma estabilização capaz de permitir um movimento preciso de punho e mão e aumentar a efetividade da realização da tarefa motora apresentada.

No presente estudo, a diferença apresentada na comparação entre os grupos dos ombros utilizados para a realização da ultrassonografia pode ser devida à fadiga muscular decorrente da postura assumida de sustentação do membro superior em abdução para a realização do exame. Associado ao tempo de realização do exame e compensações que emergem para o sistema manter-se funcional, esse desequilíbrio pode afetar a continuidade da movimentação em arcos de movimentos superiores.

Denotou-se discinesia escapular em $120^{\circ}$ de abdução, que parece ter associação direta com as dificuldades relatadas pelos participantes, como observado em alguns itens do questionário DASH Brasil relacionados a atividades cotidianas, por exemplo, "lavar as costas", "trocar uma lâmpada acima da cabeça". Para a diferença encontrada no grupo assintomático no plano frontal na posição de repouso (0), pode-se inferir que os indivíduos sintomáticos podem ter adotado uma posição antálgica durante a medida escapular, o que pode ter interferido nos ângulos encontrados entre o ombro direito e o esquerdo. Essas adaptações alte- 
ram as forças passivas e ativas que atuam no ombro durante a movimentação.

Neste estudo não houve correlação entre as variáveis analisadas. Tal fato pode dever-se a uma acomodação do indivíduo à sua atividade profissional. Deve-se ressaltar também que a avaliação objetiva nem sempre coincide com a percepção do indivíduo frente à sua incapacidade.

Portanto, alterações posturais podem comprometer a habilidade do sistema músculo-esquelético em realizar movimentos precisos e, com o tempo e com a frequência de repetição da tarefa, a dor surge como resultado desses movimentos imprecisos, o que pode acarretar comprometimento funcional, de maneira específica, dependendo da capacidade de cada indivíduo e do aparato do sistema em lidar e adaptar-se a esse novo contexto.

\section{CONCLUSÃO}

De acordo com os resultados obtidos neste estudo, foi observado que há uma relação entre a alteração da medida do ângulo de inclinação escapular e o grau de incapacidade dos membros superiores dos médicos ultrassonografistas durante o exame. A alteração da inclinação escapular dificulta a manutenção e a estabilização dos membros superiores, o que diminui sua funcionalidade durante as atividades relacionadas ao trabalho e às atividades da vida diária. O ideal é que se priorize a realização do exame de ultrassonografia no plano escapular, no qual os músculos escapulares atuam em vantagem mecânica, o que pode evitar o desequilíbrio muscular ocorrido entre esses músculos e os glenoumerais. Deve-se, também, realizar mudanças ergonômicas e enfatizar o fortalecimento da musculatura estabilizadora da escápula, com o objetivo de prevenir essas alterações e proporcionar ao médico melhor desempenho funcional na atividade ocupacional e em suas atividades cotidianas.

\section{REFERÊNCIAS}

1. Moraes GFS, Faria CDCM, Teixeira-Salmela LF. Scapular muscle recruitment patterns and isokinetic strength ratios of the shoulder rotator muscles in individuals with and without impingement syndrome. J Shoulder Elbow Surg. 2008; 17(1 Suppl):48S-53.

2. Pope DP, Croft PR, Pritchard CM, et al. Prevalence of shoulder pain in the community: the incidence of case definition. Ann Rheum Dis. 1997; 56:308-12.

3. Côté JN, Raymond D, Mathieu PA, et al. Differences in multi-joint kinematic patterns of repetitive hammering in healthy, fatigued and shoulderinjured individuals. Clin Biomech (Bristol, Avon). 2005;20:581-90.

4. Svendsen SW, Bonde JP, Mathiassen SE, et al. Work related shoulder disorders: quantitative exposure-response relations with reference to arm posture. Occup Environ Med. 2004;61:844-53.

5. Faria CDCM, Teixeira-Salmela LF, Goulart FRP, et al. Scapular muscular activity with shoulder impingement syndrome during lowering of the arms. Clin J Sports Med. 2008;18:130-6.

6. Johnson MP, McClure PW, Karduna AR. New method to assess scapular upward rotation in subjects with shoulder pathology. J Orthop Sports Phys Ther. 2001;31:81-9.

7. Cunha GM, Marchiori E, Ribeiro EJ. Avaliação ultra-sonográfica da articulação do ombro em nadadores de nível competitivo. Radiol Bras. 2007;40:403-8.

8. Downar JM, Sauers EL. Clinical measures of shoulder mobility in the professional baseball player. J Athl Train. 2005;40:23-9.
9. Wihlidal LM, Kumar S. An injury profile of practicing diagnostic medical sonographers in Alberta. Int J Ind Ergonomics. 1997;19:205-16.

10. Schoenfeld A, Goverman J, Weiss DM, et al Transducer user syndrome: an occupational hazard of the ultrasonographer. Eur J Ultrasound. 1999;10:41-5.

11. Barbosa LH, Coury HJCG. A atividade do médico ultra-sonografista apresenta riscos para o sistema músculo-esquelético? Radiol Bras. 2004;37:18791

12. David S. Importance of sonographers reporting work-related musculoskeletal injury: a qualitative view. J Diag Med Sonography. 2005;21:234-7.

13. Matias R, Pascoal AG. The unstable shoulder in arm elevation: a three-dimensional and electromyographic study in subjects with glenohumeral instability. Clin Biomech (Bristol, Avon). 2006;21(Suppl 1):S52-8.

14. Orfale AG, Araújo PMP, Ferraz MB, et al. Translation into Brazilian Portuguese, cultural adaptation and evaluation of the reliability of the disabilities of the arm, shoulder and hand questionnaire. Braz J Med Biol Res. 2005;38:293-302.

15. Dahl TH. International Classification of Functioning, Disability and Health: an introduction and discussion of its potential impact on rehabilitation services and research. J Rehab Med. 2002; 34:201-4.

16. Sampaio RF, Mancini MC, Gonçalves GGP, et al. Aplicação da Classificação Internacional de Funcionalidade, Incapacidade e de Saúde (CIF) na prática clínica do fisioterapeuta. Rev Bras Fisioter. 2005;9:129-36.

17. Jakes C. Sonographers and occupational overuse syndrome: cause, effect, and solutions. J Diag Med Sonography. 2001;17:312-20.

18. Muir M, Hrynkow P, Chase R, et al. The nature, cause, and extent of occupational musculoskeletal injuries among sonographers - recommendations for treatment and prevention. J Diag Med Sonography. 2004;20:317-25.

19. Bravo KL, Coffin CT, Murphey SL. The potential reduction in musculoskeletal injury in the nonscanning arm by using voicescan technology during sonographic examinations. J Diag Med Sonography. 2005;21:304-8. 